\title{
Multi-Atlas Segmentation of Mouse Brain MRM Based on Optimized Advanced Normalization Tools
}

\author{
Lin Lan*, Wang Jingxuan, Fu Zhenrong, Wu Xuetao, Gu Kenan, Wu Shuicai \\ College of Life Science and Bioengineering, Beijing University of Technology, Beijing, China \\ Email address: \\ lanlin@bjut.edu.cn (Lin Lan), bjutwjx@163.com (Wang Jingxuan), fuzhenrongbest@126.com (Fu Zhenrong), \\ wuxuetao@emails.bjut.edu.cn (Wu Xuetao), kenan1009@163.com (Gu Kenan), wushuicai@bjut.edu.cn (Wu Shuicai) \\ ${ }^{*}$ Corresponding author
}

\section{To cite this article:}

Lin Lan, Wang Jingxuan, Fu Zhenrong, Wu Xuetao, Gu Kenan, Wu Shuicai. Multi-Atlas Segmentation of Mouse Brain MRM Based on Optimized Advanced Normalization Tools. Science Discovery. Vol. 5, No. 6, 2017, pp. 486-491. doi: 10.11648/j.sd.20170506.26

Received: November 1, 2017; Accepted: November 24, 2017; Published: December 28, 2017

\begin{abstract}
In recent years, with the deepening of brain science research, mouse magnetic resonance microscopy (MRM) for neuroimaging research has gradually become a main research interest. Brain segmentation is an essential techniques for investigating the brain morphometry, while the traditional approach to segment a given brain involves the manual delineation of the ROIs by an expert. This practice can be slow and unscalable. Although automatic atlas-based segmentation approaches have been developed and validated for the human brain MRI, there is limited work for the mouse brain MRM. This paper combined optimized image registration and multi-atlas model for mouse brain segmentation. The results showed that multiple atlases with optimized geodesic-SyN can best improve the segmentation accuracy in the mouse brain, and registration algorithm plays important role in performance improvement.
\end{abstract}

Keywords: Mouse, Magnetic Resource Microscopy, Brain Segmentation

\section{基于优化的高等标准化工具的小鼠脑MRM图像的多图谱分割}

林岗*，王婧璇，付振荣，邬雪涛，顾克楠，吴水才

生命科学与生物工程学院, 北京工业大学, 北京, 中国

邮箱

lanlin@bjut.edu.cn（林岗）, bjutwjx@163.com（王婧璇）, fuzhenrongbest@126.com（付振荣）,

wuxuetao@emails.bjut.edu.cn (邬雪涛), kenan1009@163.com（顾克楠）, wushuicai@bjut.edu.cn（吴水才）

摘要: 近年来, 随着脑科学研究的不断深入, 基于小鼠磁共振显微成像的神经影像研究已逐渐成为该领域的研究热点 之一。大脑脑区分割是研究大脑形态学的一项重要技术，传统的分割方法一般通过专家来进行脑区的手工划分，一般 比较慢, 无法进行大量数据处理。尽管基于图谱的脑图像分割方法已被开发并在人脑MRI(Magnetic Resonance Imaging) 中得到验证,针对小鼠脑MRM图像分割的优化工作还比较有限。本文结合优化的图像配准和多图谱模型对小鼠大脑进 行分割。结果表明, 用优化的Geodesic-SyN的多图谱法取得了最好的小鼠脑组织分割精度, 其中配准算法在提高性能方 面起着极为重要的作用。

关键词：小鼠，磁共振显微成像，脑分割 


\section{1. 引言}

医学领域, 特别是脑科学领域中的重大进步都离不开 动物实验。与人体试验相比, 动物模型在缩短研究周期和 全面深入认识疾病本质等方面, 具有不可替代的地位。当 前, 表征阿尔茨海默病、帕金森氏综合征以及亨廷顿式舞 蹈症等脑疾病的小鼠模型[1-5]逐渐发展成熟, 为研究人员 提供了极大的便利。由于小鼠大脑的尺寸仅为人脑的三千 分之一，普通的临床的MRI采集设备无法进行小鼠脑图像 采集。因此, 具有更高分辨率的磁共振显微成像 (magnetic resource microscopy, MRM）[6,7]应运而生, 已成为小鼠 模型研究的重要工具, 能够帮助研究人员观察小鼠大脑细 微的内部组织结构变化。

基于感兴趣脑区的分析是小鼠MRM分析中的一种常 见的分析方式, 对脑疾病的诊断和研究具有重要意义。它 通过对小鼠脑区形状、体积等特征进行对比观察与分析, 可以挖掘出该感兴趣脑区与特定疾病之间的关系。而这种 方式的实现首先必须把感兴趣的脑区在大脑中进行标记。 手动分割方法通常被用为建立分割结果的“金标准”，一般 能得到较好的结果。而在小鼠MRM图像中, 由于组织对 比度比较低, 感兴趣区的边缘比较模糊, 实际标记一幅小 鼠脑MRM图像需要大量的时间, 而且存在效率低且可重 复性较差的问题。因此, 对动物实验中产生的大量的待标 记小鼠脑MRM图像进行手动标记基本不太现实。基于小 鼠脑图谱的先验信息, 无需人工干预的自动脑图像分割已 成为该领域的主要研究方向和发展趋势。该方法从解剖学 的角度出发, 认为不同小鼠脑MRM图像的脑结构及其位 置是相对固定和不变的。因此, 可以首先在小鼠脑图谱上 建立与图像相对应的脑区标记[11], 得到脑区先验信息; 随后, 通过图像配准算法在小鼠脑图像和脑图谱之间建立
一种一一对应的映射关系, 得到相应的变形场; 最后, 将 图谱中的脑区分割标记根据该变形场映射脑MRM图像上, 作为其对应的分割结果。

由于MRM图像中组织的对比度较低、没有清晰的分 界, 自动分割方法在实现小鼠脑组织结构的精确分割上还 存在一些问题。基于图谱的小鼠MRM脑区分割的基本处 理过程包括图像配准和标记融合两个阶段, 因此可以通过 提高配准算法的精度和进行多图谱标注信息融合对其进 行改善。图像配准是将移动图像通过变换映射到目标图像 的过程。具体到MRM图像分割中, 就是将一幅有标签的 小鼠图谱通过变换对应到需要分割的MRM图像。微分同 胚 $[8,9]$ 属于适用于微分流形范畴的同构概念, 映射及其逆 映射均为光滑。基于微分同胚的配准算法可以在保证正反 向变换一致性的情况下, 保持大脑的内部组织结构的拓扑 属性。由于微分同胚配准算法的优越性能, 它已成为脑配 准算法的主流。课题组前期已经通过对微分同肧算法进行 优化, 获得了在小鼠MRM配准中的优越结果 [10]。多图谱 标签融合问题可以假设成是一个多分类器的组合问题, 每 一个图谱可以看成一个分类器。该阶段的主要任务是将上 一阶段得到的候选标记, 根据多图谱标签融合方法对待分 割脑MRM图像中的每一个体素进行投票，最终将每个图 谱取得的分割组合起来形成一个更好, 更鲁棒的分类器。

人脑中的神经元数量是鼠脑的几千倍。基于 MRM的 鼠脑图像无论是在信噪比上还是图像分辨率上, 都和临床 的MRI图像间存在巨大的差异。针对人脑感兴趣区的多图 谱分割已有一些研究成果 $[12,13]$, 但对小鼠脑MRM图像 的自动分割进行优化的研究工作还比较少。本文主要研究 如何将优化的配准算法和多图谱标签融合来提高小鼠脑 区分割的精度。

\section{2. 材料与方法}

\section{1. 研究对象}

表1 划分的39个脑区的名字及其相应的编号。

\begin{tabular}{|c|c|c|c|}
\hline \multicolumn{2}{|c|}{ 脑区编号脑区名字 } & \multicolumn{2}{|c|}{ 脑区编号脑区名字 } \\
\hline 1 & Corpus Callosum & 21 & Entorhinal Cortex \\
\hline 2 & Lateral Ventricle & 22 & Hippocampus-CA1 Region \\
\hline 3 & Third Ventricle & 23 & Hippocampus-CA3 Region \\
\hline 4 & Cerebral Aqueduct & 24 & Dentate Gyrus \\
\hline 5 & Fourth Ventricle & 25 & General Region of Hippocampus \\
\hline 6 & Periaqueductal Gray & 26 & Superior and Inferior Colliculus \\
\hline 7 & Medulla & 27 & Pituitary \\
\hline 8 & Pons & 28 & Hypothalamus \\
\hline 9 & Cerebellar Lobules & 29 & Optic Nerve \\
\hline 10 & Cerebellar Cortex & 30 & Caudoputamen \\
\hline 11 & Anterior Commissure & 31 & General Basal Ganglia \\
\hline 12 & Lateral Olfactory Tract & 32 & The Fornix System \\
\hline 13 & Olfactory System & 33 & Septum \\
\hline 14 & Frontal Cortex & 34 & Internal Capsule \\
\hline 15 & Motor Cortex & 35 & Cerebral peduncle \\
\hline 16 & Somatosensory Cortex & 36 & SubstantiaNigra \\
\hline 17 & Auditory Cortex & 37 & Thalamus \\
\hline 19 & General Region of the Cortex & 39 & General region of the midbrain \\
\hline 20 & Perirhinal Cortex & & \\
\hline
\end{tabular}


研究中采用的MRM图像和相对应的图谱来自于新加 坡国立大学计算功能解剖实验室 (Computational Functional Anatomy Lab, CFAL ) (http://www.bioeng.nus.edu.sg/cfa/mouse_atlas.html)。该 数据包含五只 $\mathrm{C} 57 \mathrm{BL} / 6$ 雄性小鼠的 $\mathrm{T} 2$ 加权MRM图像, 采 集设备为布鲁克7-T/20-cm ClinScan系统, 图像的分辨率为 $100 * 98 * 98 \mu \mathrm{m}^{3}$ 。图像都通过 $\mathrm{N} 3$ (nonparametric non-uniform intensity normalization）进行了非均匀场校正 $[14,15]$, 然 后刚体配准到Franklin and Paxinos小鼠脑标准空间[16]进 行划分, 基于组织切片图谱将每个鼠脑划分为相互独立的 39 个解剖结构（图1）。这些人工划分的大脑感兴趣区通 常被认为是图像分割评估中的“金标准”，脑区名称及其对 应编号见表 1 。
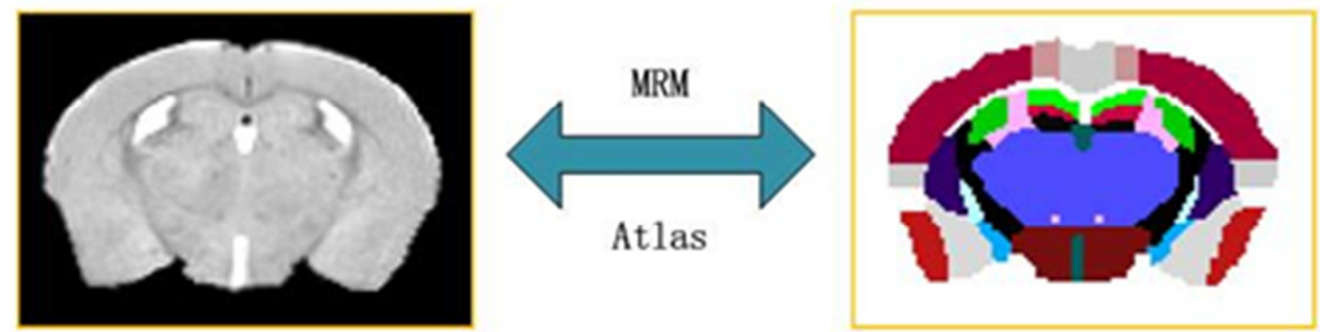

图1 小鼠脑MRM影像与对应图谱。

\section{2. 图像配准算法}

文中配准处理采用的是图像处理软件包 ANTs (https://sourceforge.net/projects/advants/) 所 包含 的 Geodesic-SyN配准算法, 在ANTs 2.x版本上运行完成。 Geodesic-SyN算法利用对称的方法将微分同胚映射 ( $\phi$ ) 分解为两个对称的映射 $\left(\phi_{1}\right.$ 和 $\left.\phi_{2}\right)$, 速度场也被分解为 两部分, 如公式1所示;

$$
\left\{\boldsymbol{v}_{1}^{*}, \boldsymbol{v}_{2}^{*}\right\}=\arg \min _{v_{1,2}}\left\{\int_{0}^{0.5}\left\|\mathrm{~L} \boldsymbol{v}_{1}(\mathrm{x}, \mathrm{t})\right\|^{2} \mathrm{dt}+\int_{0}^{0.5}\left\|\mathrm{~L} \boldsymbol{v}_{2}(\mathrm{x}, \mathrm{t})\right\|^{2} \mathrm{dt}\right.
$$

这里 $\boldsymbol{v}$ 是速度场, $\Pi_{\sim}$ 为相似性度量, $\lambda$ 为控制大脑形 变准确度的参数。 $\mathrm{L}$ 为线性算子, I和 J分别表示目标图像 和移动图像。这里 $v_{1}$ 和 $v_{2}$ 的时间范围分别为 $t \in[0,0.5]$ 和 $\mathrm{t} \in[0.5,1]$ 。该算法的优化包括依赖于时间的梯度计算以及 对微分同胚映射在每一个时间点进行更新 $\mathrm{t} \in[0,1]$, 如公式 2, 公式3所示:

$$
\begin{array}{r}
\text { Gradient: } \nabla \mathrm{E}(\mathbf{x}, \mathrm{t})=\frac{\partial \prod_{\sim}\left(\mathrm{I}\left(\phi_{1}^{-1}(\mathrm{x}, \mathrm{t})\right), \mathrm{J}\left(\phi_{2}^{-1}(\mathrm{x}, 1-\mathrm{t})\right)\right)}{\partial \phi_{\mathrm{i}}} \\
\text { Update: } \boldsymbol{v}(\mathbf{x}, \mathrm{t})=\boldsymbol{v}(\mathbf{x}, \mathrm{t})+\mathrm{G}_{\sigma} \star \nabla \mathrm{E}(\mathrm{x}, \mathrm{t})
\end{array}
$$

$\mathrm{G}_{\sigma}$ 为对速度场或变形域进行平滑的高斯滤波器。微分 同胚映射的更新是在离散域中进行的如公式4所示:

$$
\phi(\mathrm{x}, \mathrm{t}+\Delta \mathrm{t}) \leftarrow \phi(\mathrm{x}, \mathrm{t})+\Delta \mathrm{t} v(\phi(\mathbf{x}, \mathrm{t}), \mathrm{t})
$$

公式中, $\phi_{1}$ 和 $\phi_{2}$ 更新的时间范围都是 0 到 0.5 。 $\Delta \mathrm{t}$ 为离 散时间步长。对于Geodesic-SyN配准算法来说，其计算量 和内存消耗主要是因为大量的集中的梯度的计算以及微 分同胚映射的多次迭代。

改进型的互相关相似性度量通过公式5来进行计算:

$$
\mathrm{CC}(\mathbf{x})=\frac{\sum_{\mathrm{i}}\left(\left(\mathrm{I}\left(\mathrm{x}_{\mathrm{i}}\right)-\alpha_{\mathrm{I}(\mathrm{x})}\right)\left(\mathrm{J}\left(\mathrm{x}_{\mathrm{i}}\right)-\alpha_{\mathrm{J}(\mathrm{x})}\right)\right)^{2}}{\sum_{\mathrm{i}}\left(\mathrm{I}\left(\mathrm{x}_{\mathrm{i}}\right)-\alpha_{\mathrm{I}(\mathrm{x}))^{2}} \sum_{\mathrm{i}}\left(\mathrm{J}\left(\mathrm{x}_{\mathrm{i}}\right)-\alpha_{\mathrm{J}(\mathrm{x})}\right)^{2}\right.}
$$

$\alpha$ 为通过一定大小的窗口计算的局部均值, $\mathbf{x}$ 为窗口的 中心位置。随着窗口的增大, 计算量也会增大, 但是准确 性会提高。

对于研究人员来说，基于ANTs的非线性配准算法的 具体优化参数可以从四个部分进行设置, 具体优化细节详 见前期论文 $[10]$ 。表 2 为小鼠脑优化参数与人脑参数的比较。

\section{3. 多图谱融合}

多图谱方法是在单图谱分割的基础上发展而来的。简 单地说图谱就是一个模板, 包括图像和对图像的标注。配 准算法将对图谱的标注变换到目标图像上, 并将它作为目 标图像的一个图谱; 通过多图谱融合可以将得到的所有图 谱进行组合进而得到最终的脑区划分。在这里, 本研究采 用的是多数投票法, 来自于每个图谱的划分被等价看待, 将图谱中具有最多一致性的标签赋值给目标图像的体素 点。

\section{4. 实验与评估方法}

实验方法为留一法, 即每次选择 5 个图谱中的任一图 谱图像作为目标图像, 采用自动图谱分割方法通过其余图 谱得到该图像的分割结果, 再通过脑区体积重合率 (Regional volume overlap percentage, VOP) 来进行评估。 VOP表示目标像和移动像的相对应的 39 个脑区之间的体 积重合率。VOP的值定义为目标像和移动像脑区重合体积 的2倍除以目标相脑区和移动相脑区的体积之和, T和 $\mathrm{D}$ 分 别表示目标像和移动像, 计算公式为:

$$
\operatorname{VOP}(\mathrm{k})=\frac{2 \mathrm{~V}\left(\mathrm{~T}_{\mathrm{k}} \cap \mathrm{D}_{\mathrm{k}}\right)}{\mathrm{V}\left(\mathrm{T}_{\mathrm{k}}\right)+\mathrm{V}\left(\mathrm{D}_{\mathrm{k}}\right)} \times 100 \%
$$

$\mathrm{k}$ 代表39个脑区的序号, $\mathrm{V}\left(\mathrm{T}_{\mathrm{k}}\right)$ 和 $\mathrm{V}\left(\mathrm{D}_{\mathrm{k}}\right)$ 表示在 $\mathrm{k}$ 脑区目 标像 $\mathrm{T}$ 和移动像 $\mathrm{D}$ 中的体素数, $\mathrm{V}\left(\mathrm{T}_{\mathrm{k}} \cap \mathrm{D}_{\mathrm{k}}\right)$ 表示目标像和移 动像的两个脑区重合的体素数。在研究中, $\mathrm{T}_{\mathrm{k}}$ 表示目标相 中的 $\mathrm{k}$ 脑区, $\mathrm{D}_{\mathrm{k}}$ 表示移动像经过形变后得到的 $\mathrm{k}$ 脑区。VOP 值的范围为 0 到 $1 ， 1$ 表示配准精度达到 $100 \%$ 。 
3. 结果

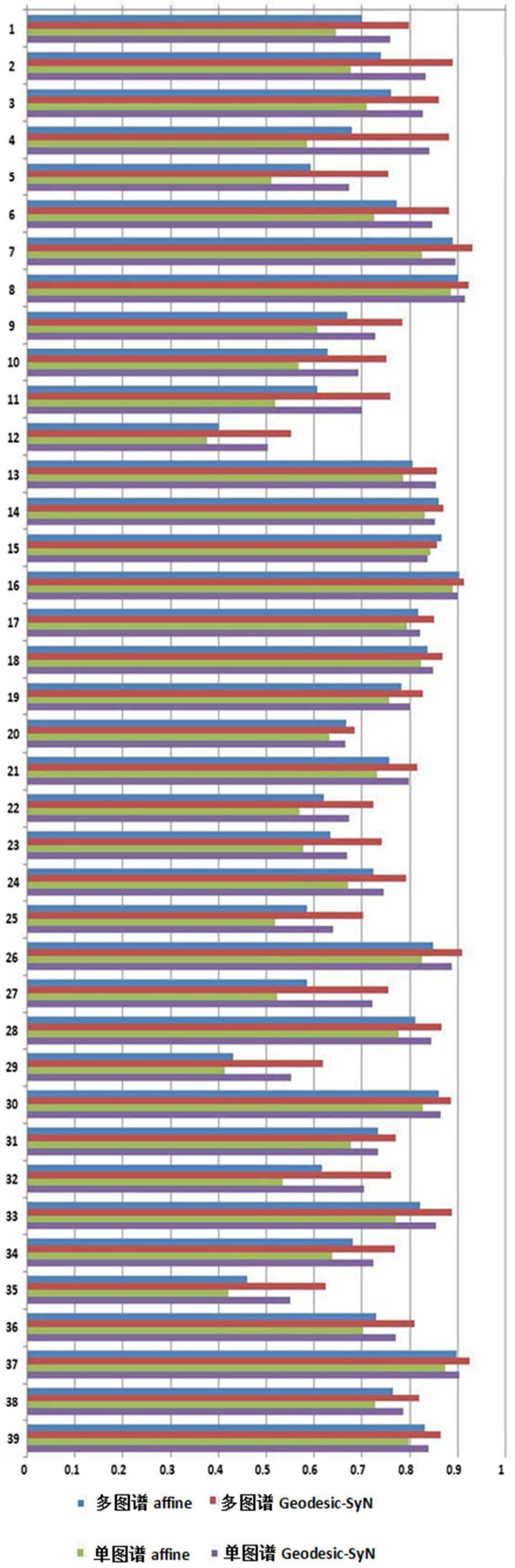

图2 四种分割算法的VOP均值, 其中横轴为VOP均值, 纵轴为对应的39 个脑区。

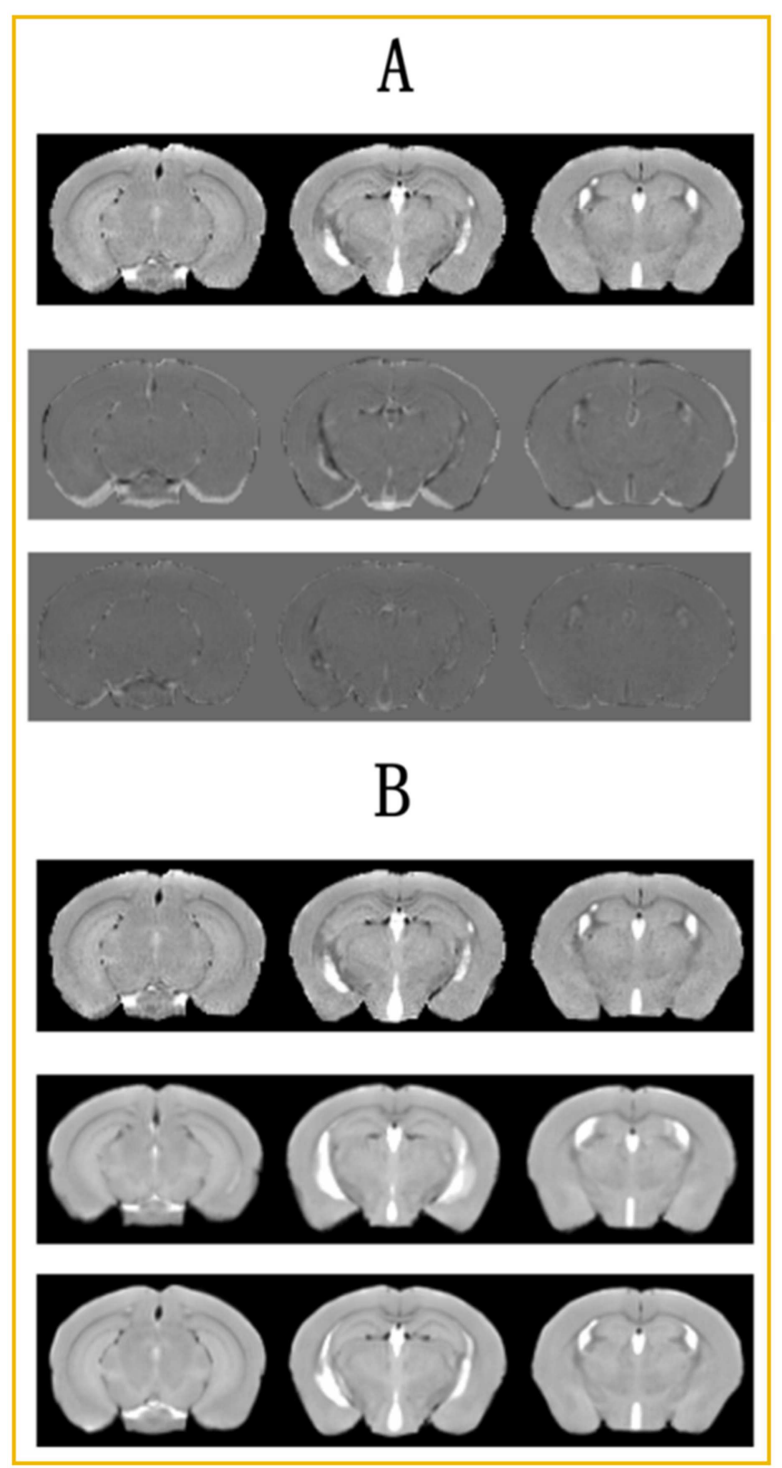

图3 单图谱affine与geodesic-SyN配准算法的灰度残差图和形变比较图 (A) 灰度残差图 (B) 形变比较图。

图2显示的是不同分割算法所获取的脑区之间VOP的 值。其中，基于多图谱Geodesic-SyN的值最高，其均值为 0.8273 , 其余几种分割算法的 VOP 值分别为: 单图谱 Geodesic-SyN (0.7913)，多图谱affine（0.73120），单图 谱 affine (0.7008)。与单图谱 affine 相比, 多图谱 Geodesic-SyN 的 VOP 值提高了 $18.05 \%$ 。和单图谱 Geodesic-SyN相比, 多图谱Geodesic-SyN算法的VOP值比 单图谱Geodesic-SyN提高了 $4.55 \%$ 。综合所有的 39 个脑区 的VOP值, 大于 $80 \%$ 的在多图谱Geodesic-SyN中占 $56.4 \%$, 在单图谱Geodesic-SyN中占 $48.7 \%$, 在多图谱affine中占 $35.9 \%$, 在单图谱affine中占 $23.1 \%$ 。在多图谱affine算法中, VOP值低于 $60 \%$ 的多达 6 个脑区; 在单图谱Geodesic-SyN 中, VOP 值低于 $60 \%$ 的也有 3 个脑区; 而在多图谱 Geodesic-SyN中, VOP值低于 $60 \%$ 的仅有 1 个脑区。多图谱 Geodesic-SyN几乎在所有的39个脑区中VOP值都是最高。 相对多图谱融合, 配准算法对图像分割的贡献更大。 
表2 小鼠MRM配准中各种标志和参数的设置。

\begin{tabular}{|c|c|c|c|c|}
\hline 主题 & 模型 & 参数 & 人脑配准优化参数 & 鼠脑配准优化参数 \\
\hline 转换模型 & Geodesic-SyN & SyN $[\Delta$, time points, DT] & $\begin{array}{l}\text { GreedyExp }[0,1] \\
\text { SyN [0.5] }\end{array}$ & $\begin{array}{l}\text { GreedyExp }[1,2] \\
\text { SyN }[0.5] \\
\text { SyN }[1,2,0.05]\end{array}$ \\
\hline 正规化 & 高斯滤波器 & Gauss $\left[\delta_{\text {gradient }}^{2}, \delta_{\text {total }}^{2}\right]$ & Gauss $[2,0]$ & Gauss $[3,2]$ \\
\hline 图像相似度 & 交叉相关 & $\begin{array}{l}\mathrm{CC} \text { [fixed image, moving image, } \\
\text { weight, radius] }\end{array}$ & PR [fixed image, moving image, 1, 2] & $\mathrm{CC}$ [fixed image, moving image, 1,4$]$ \\
\hline 图像金字塔 & 图像金字塔 & $\mathrm{I} \times \mathrm{J} \times \mathrm{K} \ldots$ & $30 \times 99 \times 11$ & $100 \times 100 \times 30$ \\
\hline
\end{tabular}

$\Delta$ 表示梯度步长, DT代表整合离散时间步长。在高斯平滑参数中, $\delta_{\text {gradient }}^{2}$ 和 $\delta_{\text {total }}^{2}$ 分别表示对速度场平滑和对变形域进行平滑, 运算方式为参数乘以图像空间的方差。Radius代表就算局部灰度值是的窗口的半径。 I, J, K表示不同分辨率下迭代的次数

图3用可视化的方法展示了单图谱在进行affine与 Geodesic-SyN配准之后的灰度残差图和形变比较图。该图 的最上面一行对应目标图像, 中间一行对应affine配准, 最下面一行对应Geodesic-SyN配准。和affine算法相比, 基 于Geodesic-SyN的配准算法得到的结果更好, 很多在affine 中形变比较大的地方都得到了很好的校正, 特别在脑区的 形状及边缘的平滑度等方面均有不小的改善。

\section{4. 结论}

实验结果表明, 配准算法的精度和多图谱分类器都对 小鼠脑MRM的分割精度存在影响。相对于多图谱分类器, 配准算法的精度对分割结果的影响更大。这显示对于图谱 分割算法，尽管多图谱在一定程度上可以改善结果，它的 核心问题还是在于如何提高配准算法的精度。另外, 虽然 本研究中采用了最为广泛使用的多数投票法, 但该方法并 没有考虑配准中个体差异对结果的影响。后期可以考虑采 用互信息来判别个体对象配准的好坏, 并以此作为权重赋 给分类器, 实现全局加权投票, 这样可以在分类器中包含 先验知识, 得到比多数投票法更优的结果。

\section{致谢}

本文为国家科技支撑计划课题（2015BAI02B03）的 阶段性成果之一。

\section{参考文献}

[1] L. Lin, Z. Fu, X. Xu, and S. Wu, "Mouse brain magnetic resonance microscopy: Applications in Alzheimer disease,"J. Microscopy Research \& Technique, 78(5)2015, pp. 416-424.

[2] S. E. Wang, and C. H. Wu, "Physiological and Histological Evaluations of the Cochlea between 3xTg-AD Mouse Model of Alzheimer's Diseases and R6/2 Mouse Model of Huntington's Diseases," J. Chinese Journal of Physiology, 58(6), 2015, pp. 359.

[3] M. Verma, D. Beaulieu-Abdelahad, G. Ait-Ghezala, R. Li, F. Crawford, M. Mullan, and D. Paris, "Chronic Anatabine Treatment Reduces Alzheimer's Disease (AD)-Like Pathology and Improves Socio-Behavioral Deficits in a Transgenic Mouse Model of AD,” J. PLOS ONE, 2015, pp. 10.
[4] M. Venissa, Z. Tanja, A. Abdelraheim, and S. Björn "Microglia-Mediated Neuroinflammation and Neurotrophic Factor-Induced Protection in the MPTP Mouse Model of Parkinson's Disease-Lessons from Transgenic Mice," J. International Journal of Molecular Sciences, 17(2), 2016, pp. 151.

[5] 林岗, 付振荣, 张柏雯, 等. PS/APP双转基因小鼠大脑中 $\beta-$ 淀粉样蛋白的磁共振显微成像和组织切片研究 [J]. 中国医 疗设备, 2016,31(2):31-33。

[6] H. BENVENISTE, and S. BLACKBAND, "MR microscopy and highresolution small animal MRI: Applications in neuroscienceresearch,” J. Prog Neurobiol, 67(5), 2002, pp. 393-420.

[7] B. DRIEHUYS, J. NOULS, A. BADEA, E. Bucholz, K. Ghaghada, A. Petiet, and L. W. Hedlund, "Small animal imaging withmagnetic resonance microscopy," J. ILAR J, 49(1), 2008, pp. 35-53.

[8] B. B. Avants, C. L. Epstein, M Grossman, and J. C. Gee, "Symmetric Diffeomorphic Image Registration with Cross-Correlation: Evaluating Automated Labeling of Elderly and Neurodegenerative Brain," J. Medical Image Analysis, 12(1), 2008, pp. 26-41.

[9] B. B. Avants, N. J. Tustison, G. Song, P. A. Cook, A. Klein, and J. C. Gee, "A reproducible evaluation of ANTs similarity metric performance in brain image registration,"J. Neuroimage, 54(3), 2011, pp. 2033-2044.

[10] Z. Fu, L. Lin, M. Tian, J. Wang, B. Zhang, P. Chu, S. Li, M. M. Pathan, Y. Deng, and S. Wu,"Evaluation of five diffeomorphic image registration algorithms for mouse brain magnetic resonance microscopy," J. Microsc,268(2),2017, pp. 141-154.

[11] 付振荣, 林岗, 张柏雯, 等. 基于 MRM的小鼠脑模板创建 的研究进展 [J]. 中国医疗设备, 2016, 31(2): 25-30。

[12] A. Gholipour, A. Akhondiasl, J. A. Estroff and S. K. Warfield, "Multi-atlas multi-shape segmentation of fetal brain MRI for volumetric and morphometric analysis of ventriculomegaly,"J. Neuroimage, 2012 15; 60(3): 1819-31.

[13] J. E. Iglesias and M. R. Sabuncu,"Multi-atlas segmentation of biomedical images: A survey,"J. Med Image Anal,24(1), pp. 205-219, Aug 2015.

[14] L. Lin, S. Wu, G. Bin, and C. Yang, "Intensity Inhomogeneity Correction Using N3 on Mouse Brain,"J. Magnetic Resonance Microscopy [J]. Journal of Neuroimaging Official Journal of the American Society of Neuroimaging, 23(4),2013, pp. 502-507. 
[15] J. G. Sled, A. P. Zijdenbos, and A. C. Evans, "A nonparametric method for automatic correction of intensity nonuniformity in MRI data," J. IEEE Transactions on Medical Imaging, 17(1), 1998, pp. 87-97.
[16] K. B. J. Franklin, G. Paxinos, The Mouse Brain: In Stereotaxic Coordinates, 1st ed., New York: Academic Press, 2001. 